CORRESPONDENCE

\section{Can microsatellite data allow identification of oleaster Plio-Pleistocene refuge zones in the Mediterranean Basin?}

A response to Breton, C., Tersac, M. \& Bervillé, A. (2006) Genetic diversity and gene flow between the wild olive (oleaster, Olea europaea L.) and the olive: several PlioPleistocene refuge zones in the Mediterranean basin suggested by simple sequence repeats analysis. Journal of Biogeography, 33, 1916-1928.

In a recent paper, Breton et al. (2006) provided interesting genetic data (mainly nuclear microsatellite loci) to investigate the post-glacial history of oleaster populations in the Mediterranean Basin. They analysed the population genetic structure using a model-based Bayesian procedure implemented in STRUCTURE (Pritchard et al., 2000). Based on this methodology, putative reconstructed panmictic oleaster populations (RPOPs) were identified. Seven RPOPs were thus recognized but about one-third of individuals (59/166) were not assigned to one of these groups. These individuals were regarded as probably issued from hybridizations between trees from different populations. The RPOPs were hypothesized to possibly overlay glacial refuges. This proposal is quite speculative. Indeed, the genetic differentiation between populations can be influenced by several parameters (such as genetic drift and isolation) and may be shaped over a relatively small period of time even though olive trees are known to have a long life span. In illustration, we studied two distant populations of invasive olive (Olea europaea subsp. cuspidata) from east Australia (Campbelltown) and Hawaii, USA (Maui). In a previous study, we characterized the plastid and ribosomal DNA lineages of 14 wild populations of O. europaea subsp. cuspidata distributed from South Africa to China (Besnard et al., in press). In invasive populations, the same plastid and ribosomal DNA lineages characteristic of South African populations were revealed. This indicates that these invasive trees of O. europaea subsp. cuspidata originated from southern Africa. In addition, seven nuclear microsatellite loci were used to characterize the two invasive populations. Among the 16 microsatellite alleles detected in the trees of Maui, 15 were also detected at Campbelltown (in which 40 alleles were detected), highlighting the great genetic proximity between these two populations (Besnard et al., unpublished data). Based on this evidence, east Australian and Hawaiian invasive populations were considered to have originated from the same genetic pool during the last 200 years (Spennemann \& Allen, 2000). Moreover, the reduced genetic diversity in Hawaii supports the possibility that eastern Australia could have been an intermediate source for the introduction to Hawaii as indicated by historical records (Spennemann \& Allen, 2000; Starr et al., 2003). To analyse microsatellite data, a model-based Bayesian procedure was used following Breton et al. (2006) which allowed us to identify two clusters corresponding to Maui (cluster 1) and Campbelltown (cluster 2; Fig. 1). All individuals were assigned with probabilities greater than the minimum threshold of $80 \%$ used by Breton et al. (2006). This general pattern clearly indicates that genetic differentiation between populations can rapidly appear during colonization due to founder effects, genetic drift and isolation. However, following Breton et al. (2006), these clusters would have been interpreted as potential relict populations. Such a conclusion would obviously be misleading since Maui and Campbelltown are recent populations. Furthermore, in the Mediterranean Basin, oleaster populations are distributed in a heterogeneous habitat. For instance, populations are present on all main Mediterranean islands and may then be isolated from each other. In such conditions, we can expect that genetic drift and founder effects should contribute to increase the genetic differentiation between populations as reported for the invasive olives. In addition, important displacement of cultivars (landraces or clones) by humans has occurred during the last millennia and gene flow from orchards to the wild may have greatly influenced the genetic structure of the present oleaster populations (Breton et al., 2006). We can expect that, after hybridization between distant populations, panmictic equilibrium can be attained creating new RPOPs. Nevertheless, the long generation time of olive trees coupled with recurrent gene flow from successively introduced cultivars may have prevented populations from attaining such equilibrium, as attested by the numerous individuals not clearly assigned to a RPOP in Breton et al. (2006). Lastly, the history of cultivar introduction should be different in each Mediterranean area, probably contributing to the complexity of the general pattern. For instance in Breton et al. (2006), Corsican populations belong to four of the six proposed genetic groups. All these features make genetic data of wild olive relatives challenging to interpret in a straightforward way. In particular, they may not allow a clear identification of refuge zones as is possible for forest trees for which human influence has been less important (e.g. Magri et al., 2006). We can thus conclude that the use of nuclear microsatellite loci may be insufficient to detect oleaster Plio-Pleistocene refuges in the Mediterranean Basin and that comparative analyses of fossil, phylogenetic and genetic data (and particularly the genetic characterization of archaeological material; Elbaum et al., 2006) are required in order to reconstruct patterns of oleaster post glacial recolonization in the Mediterranean Basin. 
(a)

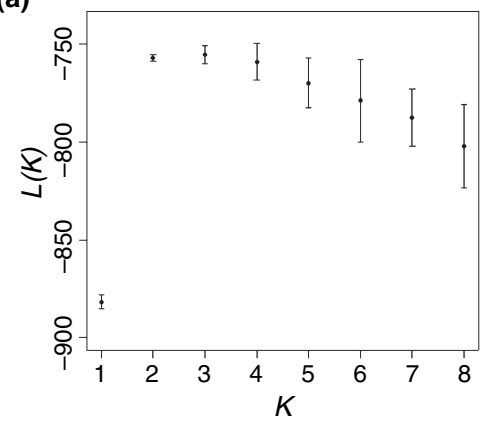

(b)

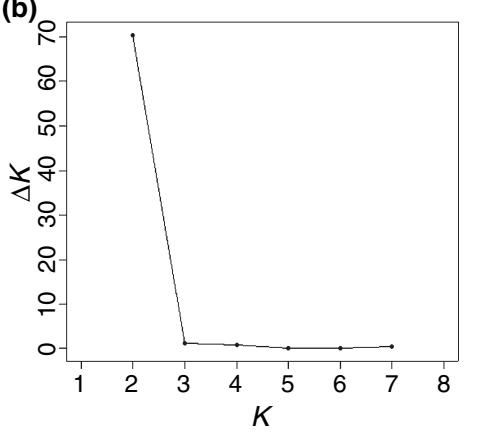

(c)

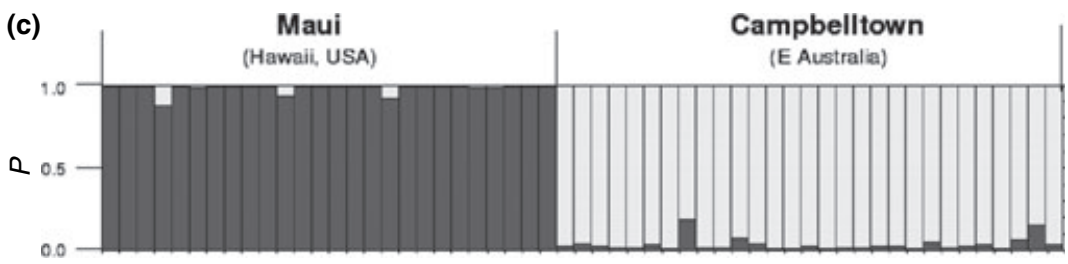

Figure 1 Population structure assessed using Bayesian inference (Pritchard et al., 2000) for two invasive olive populations (55 individuals). These analyses are based on seven nuclear microsatellite loci [i.e. DCA1, DCA3, DCA8, DCA9, DCA14, EMO3 and PA(ATT)2] rendering 41 alleles. Run parameters were identical to those used by Breton et al. (2006). Criteria used to determine the most likely number of clusters $(K)$ were the highest rate of change of the likelihood distribution together with the smallest variance over the iterations following Evanno et al. (2005). (a) Mean likelihood $[L(K)]$ ( \pm standard deviation) over 10 runs for each $K$ value. The most important increase in likelihood value is at $K=2$. This $K$ is also the one that exhibits the lowest variance. (b) $\Delta K$ calculated as the mean of the absolute values of the second-order rate of change of $L(K)$ with respect to $K$ divided by the standard deviation of $L(K)$ over 10 runs. The highest value of $\Delta K$ is for $K=$ 2. This is then the most likely number of clusters. (c) Probability $(P)$ of the different individuals (represented by vertical bars) being assigned to each of the two clusters (cluster 1 in dark grey and cluster 2 in light grey) averaged over 10 runs. Whether the olive trees belong to Maui or Campbelltown populations is indicated. On average, individuals from Maui were assigned at $98 \%$ (standard deviation = 3) to cluster 1 and individuals from Campbelltown at $97 \%$ (standard deviation $=4$ ) to cluster 2 .

G. Besnard, L. Wille, P. Henry, E. Chapuis \& P. A. Christin

Department of Ecology and Evolution, Biophore, University of Lausanne, 1015 Lausanne, Switzerland. E-mail: gbesnard@unil.ch

\section{REFERENCES}

Besnard, G., Rubio de Casas, R. \& Vargas, P. (in press) Plastid and nuclear DNA polymorphism reveals historical processes of isolation and reticulation in the olive tree complex (Olea europaea L.). Journal of Biogeography doi:10.1111/j.1365-2699. 2006.01653.x.

Breton, C., Tersac, M. \& Bervillé, A. (2006) Genetic diversity and gene flow between the wild olive (oleaster, Olea europaea L.) and the olive: several Plio-Pleistocene refuge zones in the Mediterranean basin suggested by simple sequence repeats analysis. Journal of Biogeography, 33, 1916-1928.
Elbaum, R., Melamed-Bessudo, C., Boaretto, E., Galili, E., Lev-Yadun, S., Levy, A.A. \& Weiner, S. (2006) Ancient olive DNA in pits: preservation, amplification and sequence analysis. Journal of Archaeological Science, 33, 77-88.

Evanno, G., Regnaut, S. \& Goudet, J. (2005) Detecting the number of clusters of individuals using the software STRUCTURE: a simulation study. Molecular Ecology, 14, 2611-2620.

Magri, D., Vendramin, G.G., Comps, B., Dupanloup, I., Geburek, T., Gomory, D., Latalowa, M., Litt, T., Paule, L., Roure, J.M., Tantau, I., Van der knaap, W.O., Petit, R.J. \& de Beaulieu, J.L. (2006) A new scenario for the Quaternary history of European beech populations: palaeobotanical evidence and genetic consequences. New Phytologist, 171, 199-221.

Pritchard, J.K., Stephens, M. \& Donnelly, P. (2000) Inference of population structure from multilocus genotype data. Genetics, 155, 945-959.

Spennemann, D.H.R. \& Allen, L.R. (2000) Feral olives (Olea europaea) as future woody weeds in Australia: a review. Australian Journal of Experimental Agriculture, 40, 889-901.

Starr, F., Starr, K. \& Loope, L. (2003) Plants of Hawaii. http://www.hear.org/starr/ hiplants/reports/index.html.

Editor: Robert Whittaker 